\title{
Citations
}

\section{Delivery Included}

A critical factor for dictating gene silencing efficiency is how readily a short interfering RNAs (siRNA) can be delivered into cells. A variety of nanocarriers have been tested, including liposomes, polymer complexes, and self-assembling nanoparticles. Unfortunately, these diverse approaches have been hampered by an equally lengthy list of limitations, including complicated syntheses and poor encapsulation efficiencies. In a creative workaround appearing in Nature Materials, Lee et al. have proposed using the cargo itself as the carrier: instead of encapsulating siRNA within a delivery vehicle, the structure is formed from the RNA that will do the silencing. Nanofabrication of the combined cargo-carrier begins with circularizing a 92-nucleotide DNA oligonucleotide by hybridizing it to a shorter oligonucleotide that bridges the two ends. As the double-stranded region forms a T7 promoter sequence, and the siRNA coding sequences are found in the singlestranded region of the circle, rolling circle transcription creates multiple tandem repeats of the RNA hairpin. Over time, the tangled strands form a fiber, then a sheet, and then pucker into spherical structures the authors call "RNAi microsponges" because of the "pores" formed by the intertwined RNA sheets. Each of these remarkable structures contains a half million hairpins in a crystalline structure that is relatively impervious to temperatures up to $150^{\circ} \mathrm{C}$ but is accessible to Dicer, releasing over 100,000 siRNA copies per microsponge in in vitro studies. For delivery into cells, Lee et al. condensed the microsponges with polyethylenimine, a polycation that has been previously used for siRNA

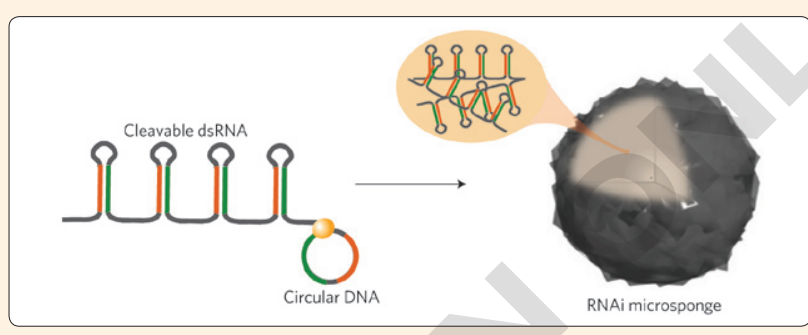

Self-assembly of RNAi microsponge from rolling circle transcription of RNA hairpin sequences. Source: Nature Materials

transfection. With this treatment, the microsponges shrink to a tenth of their original size, collapsing the porous structure. When tested in tissue culture, the RNAi microsponges reduced target gene expression to a greater degree than siRNA delivered by polyethylenimine; similarly, significant decreases in luciferase signal were evident following injection into mice with luciferaseexpressing tumors. A striking benefit of the new delivery system is that 1,000-fold less carrier was needed for silencing than when using a conventional delivery vehicle. These results provide initial validation of the cargo-as-carrier approach, delivering some good news to one of the most vexing limitations of RNAi.

\section{Lee et al. Self-assembled RNA interference microsponges for efficient siRNA delivery. Nat Mater. [Epub ahead of print, February 26, 2012; doi: 10.1038/NMAT3253].}

\section{Under Development}

Techniques for expression profiling and epigenetic characterization have matured sufficiently to provide a wealth of data on how cells function, yet strategies for monitoring changes in a developing organism are less evolved. A number of cell type-specific RNA isolation methods enable expression profiling, but isolating the chromatin needed for epigenetic analysis remains problematic. In principle, flow cytometric sorting or laser capture microdissection might do the trick, but in practice suffer from their complexity and relatively low yields of chromatin. In an article in Genome Research, Steiner et al. advocate a method based upon cell typespecific purification of nuclei. In previous work, the author had introduced INTACT (isolation of nuclei tagged in specific cell types) for use in the Arabidopsis root epidermis. That plant tissue comprises just two cell types, so it was debatable whether the approach could be adapted to more complex animal models. Steiner et al. tackled the question using $C$. elegans and Drosophila. In both cases, they fused a sequence that is a bacterial biotin ligase substrate to a protein that is targeted to the nuclear envelope. This nuclear targeting fusion (NTF) was expressed under a cell type- or tissue-specific promoter. Transgenic animals expressing both the NTF and the biotin ligase were used for nuclei isolation. In studies involving worms, adult animals were fixed, homogenized, and nuclei isolated by streptavidin affinity purification. Though muscle cells - the target in these experiments-represent just $5 \%$ of total cells in the adult worm, their nuclei were isolated at more than $90 \%$ purity. In Drosophila, the target was embryonic mesoderm, for which $0.5-1 \mathrm{~g}$ of embryo starting material suffice for nuclei isolation at comparable levels of purity. Since nuclear RNA has been shown to correlate with the total cellular pool, these preparations could be used for expression profiling of the target tissue. The genes picked up in these analyses compared favorably with lists of tissue-specific genes generated by more labor-intensive methods, but the unique advantage of the new method is the information it provides about the chromatin landscape. For the nuclei purified from $C$. elegans, the authors performed nucleosome occupancy profiling, confirming for the first time in animals the in vitro finding that highly expressed genes have low nucleosome occupancy. These results show that simultaneous transcriptional and epigenetic characterization of specific tissues is now within reach, which can be expected to rapidly build a fuller understanding of developmental pathways.

Steiner et al. Cell-type-specific nuclei purification from whole animals for genome-wide expression and chromatin profiling. Genome Res. [Epub ahead of print, February 8, 2012; doi:10.1101/ gr.131748.111].

Selected and written by Nijsje Dorman, Ph.D. [C]A]

BioTechniques 52:295 (May 2012)

doi 10.2144/000113845

To purchase reprints of this article, contact:

biotechniques@fosterprinting.com 